\title{
Influence of laboratory reports on prescribing of antimicrobials for urinary tract infection
}

\author{
MP BARNES \\ From Bristol Royal Infirmary, Bristol BS2 $8 \mathrm{HW}, \mathrm{UK}$
}

SUMMARY The effect of laboratory reports, with restricted reporting of antibiotic information, on the prescribing of antimicrobials for urinary tract infection was assessed in a series of 250 patients. There was considerable prescribing of broad-spectrum antimicrobials, to which sensitivity was rarely reported, whether treatment was begun before or after the laboratory report was available, although they were used rather less in the latter situation. Of 147 patients with no evidence of bacteriuria, $26(18 \%)$ received a full course of antimicrobial treatment. Of 49 asymptomatic catheterised patients with bacteriuria, $30(61 \%)$ received antibiotics despite a recommendation to the contrary. The influence of laboratory reports is limited, and when the emergence and spread of resistant bacteria necessitates restraint with antimicrobials, restriction of antibiotic sensitivity reporting and recommendations by the laboratory may be insufficient.

Several surveys have illustrated the widespread misuse of antibiotics in hospital practice, ${ }^{1-4}$ and the overuse of broad-spectrum antibiotics has been shown to encourage the emergence of resistant bacteria. ${ }^{5}$ In the Bristol Health District, between February 1976 and March 1978, over 300 patients were colonised by a multiresistant strain of Klebsiella aerogenes, primarily involving the urinary tract, and it was established that the use of broad-spectrum antibiotics, particularly co-trimoxazole, encouraged such colonisation. ${ }^{6}$

This outbreak, coupled with the emergence of resistance to the newer antibiotics in staphylococci and in Gram-negative bacilli6 7 necessitated the introduction of a more restrictive antibiotic policy in the district than had been used previously.

This survey was designed to determine how much the laboratory reports, which incorporated the new policy recommendations, could affect the prescribing of antimicrobials. It was limited to urinary tract infections, in which the criteria for infection and treatment are rather more definable than in many other situations.

\section{Administration of the policy}

Three approaches were adopted: continued educational efforts; restriction by the pharmacy of the issue of certain newer antimicrobials until discussion

Received for publication 31 October 1979 between the clinician and a microbiologist had taken place; and limitation of the reporting of antibiotic sensitivity results by the laboratory.

\section{Methods}

PROCESSING OF URINE SPECIMENS

All urine specimens are submitted to microscopy. Specimens showing abnormalities (cells or bacteria) have a Gram-film examination and receive semiquantitative culture by the standard loop method, and 'direct sensitivity' to sulphonamide, trimethoprim, ampicillin, nitrofurantoin, nalidixic acid, and gentamicin is established. The remainder are cultured by the paper strip method. Sensitivity to other antimicrobials is tested later only for multiresistant isolates and for certain bacterial species, such as staphylococci and Pseudomonas aeruginosa.

REPORTING OF ANTIBIOTIC SENSITIVITIES

Sensitivities are reported only on specimens thought to show significant bacteriuria, usually established as more than $10^{5}$ colonies per $\mathrm{ml}$ in mid-stream specimens. All sensitivities are reported on the laboratory copy. Sensitivity to sulphonamide and nitrofurantoin is reported to the ward; only if there is resistance to both of these drugs is sensitivity to a further agent reported-in descending order of preference, ampicillin, co-trimoxazole, nalidixic acid, and gentamicin, with cephalosporins and other agents as second-line drugs. All resistance is also reported to the ward. A 'recommended antibiotic', 
usually sulphonamide or nitrofurantoin, is also given. Treatment is discouraged for Streptococcus faecalis and Staphylococcus epidermidis bacteriuria, and for asymptomatic patients with indwelling catheters, unless instrumentation is contemplated or there are other risk factors.

\section{Survey}

During nine weeks all first urine specimens from inpatients at Bristol Royal Infirmary were considered. Patients were not included if they were already receiving an antimicrobial for another reason.

The patients included were seen on the ward the same day, and the clinical information was recorded. On the next day the laboratory results were noted, and the report was returned to the ward in the usual way. One or two days later the patient was again seen on the ward, and any changes in symptoms, treatment, catheter, etc, were recorded. Each patient was followed until the course of treatment was complete or he was discharged from hospital.

\section{Results}

The survey included 250 specimens, 132 from females and 118 from males. In 103 specimens there was considered to be significant growth, and sensitivities as described above were sent to the ward. In 147 specimens there was no growth or insignificant growth and no sensitivities were recorded.

Forty-eight patients were treated with antibiotics before the laboratory result was known. Twentyseven of these were subsequently shown to have significant bacteriuria, and all continued with their original prescription. In five, however, the bacterium isolated was 'resistant' to the antibiotic being used. The remaining 21 patients proved to have no evidence of bacteriuria, but in only three of these was the antibiotic stopped, the remaining 18 completing the course of treatment, in most cases with an unfavoured antimicrobial such as co-trimoxazole.

A further 48 patients were started on antibiotic therapy after the laboratory report had been received. Of these 48 patients, only 20 were treated according to the recommendation given on the report; most discrepancies were in the use of co-trimoxazole where only sulphonamide had been recommended. The choice of antimicrobials, with and without benefit of the laboratory results, is compared in the Figure. Considerable prescribing of co-trimoxazole and ampicillin or amoxycillin occurred in both groups. The effect of the recommendation of sulphonamide and of nitrofurantoin can also be seen.

In the total series, 81 patients had catheters in situ

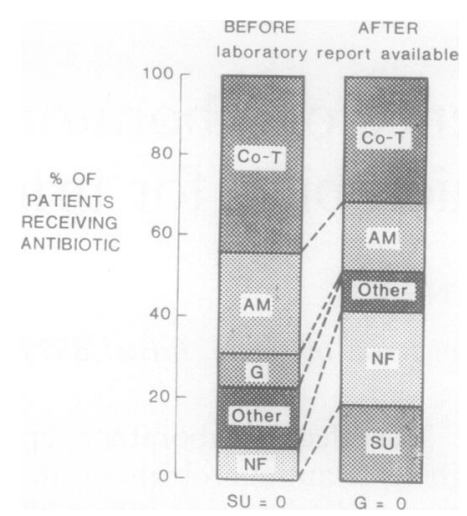

Choice of antimicrobial, with and without availability of laboratory report: $C o-T=$ co-trimoxazole; $A M=$ ampicillin or amoxycillin; $G=$ gentamicin; $S U=$ sulphonamide; $N F=$ nitrofurantoin; 'Other' includes cephalosporins, tetracycline, carfecillin, and nalidixic acid.

(Table 1). Of these, 52 showed evidence of bacteriuria and $33(63 \%)$ were treated with a course of antibiotics. All but three of these colonised patients were asymptomatic, and in none was instrumentation of the urinary tract contemplated. Fourteen of the 29 patients with no evidence of bacteriuria were also treated.

Table 1 Treatment in catheterised patients

\begin{tabular}{lll}
\hline & $\begin{array}{l}\text { 'Significant' } \\
\text { growth }\end{array}$ & $\begin{array}{l}\text { No 'significant' } \\
\text { growth }\end{array}$ \\
\hline Antibiotic course & & \\
prescribed & 33 & 14 \\
No treatment given & 19 & 15 \\
Total & 52 & 29 \\
\hline
\end{tabular}

Treatment in the non-catheterised group is shown in Table 2. Twelve of the 118 people with no significant growth were nevertheless given a course of treatment. Conversely, 17 patients with proven infection were not treated. Six of these had Strep. faecalis bacteriuria, one yielded Candida albicans in heavy growth, and two cases were reported as of 'borderline significance' but sensitivities were reported to the ward. In the remaining eight cases, there was significant bacteriuria with Gram-negative bacilli of potential pathogenicity.

In the total series there were 67 cases of bacteriuria that were treated with antimicrobials. In 33 cases no follow-up specimen was received. Analysis of the 34 follow-up specimens received showed that in 17 there was now no growth. Six of these patients were catheterised. In nine cases the original bacterium apparently persisted; five patients in this group were 
Table 2 Treatment in non-catheterised patients

\begin{tabular}{lll}
\hline & $\begin{array}{l}\text { 'Significant' } \\
\text { growth }\end{array}$ & $\begin{array}{l}\text { No 'significant' } \\
\text { growth }\end{array}$ \\
\hline Antibiotic course & & \\
prescribed & 34 & 12 \\
No treatment given & 17 & 106 \\
Total & 51 & 118 \\
\hline
\end{tabular}

catheterised. In the remaining eight cases a new strain of bacterium was grown; six of these eight were catheterised. Seven of the eight had originally lactose-fermenting coliforms and one a Proieus species. These were replaced, after treatment, by more resistant lactose-fermenting coliforms (4) and Proteus species (1), and by Strep. faecalis (2) and Ps. aeruginosa (1).

\section{Discussion}

In a survey of this kind it is difficult to generalise and easy to criticise the treatment of individual patients, and the results should be interpreted with some degree of caution.

The use of any restrictive policy is controversial. It imposes epidemiological considerations on the selection of the best agent for the individual patient, and it is not necessarily effective in removing resistant bacteria from the environment. ${ }^{8}$ An unsuitable antibiotic may be recommended by the laboratory for a particular patient, for example, nitrofurantoin in renal failure. The policy must be flexible enough to cope with future changes in the local situation. Despite these criticisms several authors have reported success with restrictive policies. ${ }^{9} 10$

The results of this survey show, however, that the present approach has met with only limited success in influencing treatment, at least with respect to urinary infection. It was disturbing to find such a range of broad-spectrum prescribing. It is appreciated that treatment may have to be initiated before the laboratory report is received, but in many cases an unsuitable initial choice of treatment was not changed after the result and laboratory recommendations had been received.

The recommendation to withhold treatment in asymptomatic, catheterised patients with bacteriuria is more questionable, but it is felt that such treatment is often fruitless in patients with long-standing indwelling catheters. It is of interest that in six catheterised patients a new, more resistant bacterium was grown after a course of treatment; and it was in such patients that the first cases of colonisation by the resistant $K$. aerogenes were detected. ${ }^{6}$

It seems that manipulation of laboratory reports and general policy exhortations are insufficient to alter prescribing sufficiently. It may be that more rigid and formal restriction and a more intensive educational approach are necessary.

I am grateful to Professor DCE Speller for advice during this investigation.

\section{References}

1 Achong MR, Wood J, Theal HK, Goldberg R, Thompson DA. Changes in hospital antibiotic therapy after a quality-of-use study. Lancet 1977; 2:1118-22.

${ }^{2}$ Castle M, Wilfert CM, Cate TR, Osterhout S. Antibiotic use at Duke University Medical Center. JAMA 1977;237:2819-22.

${ }^{3}$ Scheckler WE, Bennett JV. Antibiotic usage in seven community hospitals. JAMA 1970;213:264-7.

${ }^{4}$ Roberts AW, Visconti JA. The rational and irrational use of systemic antimicrobial drugs. Am J Hosp Pharm 1972;29:828-34.

${ }^{5}$ Finland M. Changing ecology of bacterial infections as related to antibacterial therapy. J Infect Dis 1970; 122:419-31.

${ }^{6}$ Curie K, Speller DCE, Simpson RA, Stephens M, Cooke DI. A hospital epidemic caused by a gentamicin-resistant Klebsiella aerogenes. J Hyg (Camb) 1978;80:115-23.

7 Speller DCE, Raghunath D, Stephens M et al. Epidemic infection by a gentamicin-resistant Staphylococcus aureus in three hospitals. Lancet 1976;1:464-6.

${ }^{8}$ Bridges K, Kidson A, Lowbury EJL, Wilkins MD. Gentamicin- and silver-resistant pseudomonas in a burns unit. $B r$ Med J 1979;1:446-9.

${ }^{9}$ Price DJE, Sleigh JD. Control of infection due to Klebsiella aerogenes in a neurosurgical unit by withdrawal of all antibiotics. Lancet 1970;2:1213-15.

10 McGowan JE, Jr, Finland M. Usage of antibiotics in a general hospital; effect of requiring justification. J Infect Dis 1974;130:165-8.

Requests for reprints to: Dr M P Barnes, 33 Summertrees Avenue, Greasby, Wirral, Merseyside, UK. 